\title{
On the Rotation of Supermassive Stars
}

\author{
Lionel Haemmerlé $^{1}$ (D), Tyrone E. Woods ${ }^{2}$, Ralf S. Klessen ${ }^{3,4}$ (D), Alexander Heger ${ }^{2}$ (D), and Daniel J. Whalen ${ }^{5}$ (DD \\ ${ }^{1}$ Observatoire de Genève, Université de Genève, chemin des Maillettes 51, CH-1290 Sauverny, Switzerland; lionel.haemmerle@unige.ch \\ ${ }^{2}$ Monash Centre for Astrophysics, School of Physics and Astronomy, Monash University, VIC 3800, Australia \\ ${ }^{3}$ Universität Heidelberg, Zentrum für Astronomie, Institut für Theoretische Astrophysik, Albert-Ueberle-Str. 2, D-69120 Heidelberg, Germany \\ ${ }^{4}$ Universität Heidelberg, Interdisziplinäres Zentrum für Wissenschaftliches Rechnen, Im Neuenheimer Feld 205, D-69120 Heidelberg, Germany \\ 5 Institute of Cosmology and Gravitation, University of Portsmouth, Dennis Sciama Building, Portsmouth PO1 3FX, UK \\ Received 2017 November 27; revised 2017 December 27; accepted 2017 December 27; published 2018 January 16
}

\begin{abstract}
Supermassive stars (SMSs) born from pristine gas in atomically cooled halos are thought to be the progenitors of supermassive black holes at high redshifts. However, the way they accrete their mass is still an unsolved problem. In particular, for accretion to proceed, a large amount of angular momentum has to be extracted from the collapsing gas. Here, we investigate the constraints stellar evolution imposes on this angular momentum problem. We present an evolution model of a supermassive Population III star simultaneously including accretion and rotation. We find that, for SMSs to form by accretion, the accreted angular momentum has to be about $1 \%$ of the Keplerian angular momentum. This tight constraint comes from the $\Omega \Gamma$ limit, at which the combination of radiation pressure and centrifugal force cancels gravity. It implies that SMSs are slow rotators, with a surface velocity less than 10\%-20\% of their first critical velocity, at which the centrifugal force alone cancels gravity. At such low velocities, the deformation of the star due to rotation is negligible.
\end{abstract}

Key words: stars: massive - stars: rotation

\section{Introduction}

Supermassive stars (SMSs), with masses $M>10^{4} M_{\odot}$, are candidates for the progenitors of supermassive black holes at high redshifts (e.g., Bromm \& Loeb 2003; Begelman et al. 2006; Regan \& Haehnelt 2009; Agarwal et al. 2012, 2017; Latif et al. 2013a, 2013b; Dijkstra et al. 2014; Inayoshi et al. 2014; Regan et al. 2014). In this scenario, a primordial halo is devoid of $\mathrm{H}_{2}$ molecules due to a strong external Lyman-Werner radiation field from nearby star-forming regions. Without this cooling agent, the temperature rises above $\sim 10^{4} \mathrm{~K}$, preventing star formation before the halo's mass reaches $\sim 10^{7}-10^{8} M_{\odot}$. At this point, the collapse is triggered at rates of $\sim 1 M_{\odot} \mathrm{yr}^{-1}$, toward a central stellar object (Latif et al. 2013b; Becerra et al. 2015; Smidt et al. 2017). Stellar evolution models of Population III (Pop III) SMSs show that protostars accreting at these high rates evolve as red supergiants along the Hayashi limit, with an inflated envelope and a low surface temperature, thus keeping a weak ionizing feedback on the accretion flow (Hosokawa et al. 2012, 2013; Haemmerlé et al. 2018). This allows us to maintain accretion onto the protostar toward stellar masses in the supermassive range (Hirano et al. 2017). The star is thought to accrete until $M \simeq 3 \times 10^{5} M_{\odot}$, before collapsing into a black hole (Umeda et al. 2016; Woods et al. 2017; Haemmerlé et al. 2018) due to the general relativistic (GR) instability (Chandrasekhar 1964). Classical pulsational instabilities have been shown not to prevent the star from growing by accretion until this stage (Hosokawa et al. 2013; Inayoshi et al. 2013a, 2013b).

Star formation requires processes that remove angular momentum from the inner regions of collapsing prestellar clouds, otherwise the centrifugal force would overcome gravity and prevent further collapse (Spitzer 1978; Bodenheimer 1995; Maeder 2009). Numerical studies of Pop III star formation indicate that primordial protostellar seeds rotate near their critical limit (Stacy et al. 2011, 2013), at which the centrifugal force exceeds the gravitational attraction. Above this limit, hydrostatic equilibrium cannot be achieved and the star breaks up. The critical limit is given by

$$
\frac{\mathrm{v}_{\text {crit }, 1}^{2}}{R_{\text {eq, crit }}}=\frac{G M}{R_{\text {eq, crit }}^{2}} \quad \Longrightarrow \quad v_{\text {crit }, 1}=\sqrt{\frac{G M}{R_{\text {eq, crit }}}},
$$

where $R_{\text {eq,crit }}$ is the equatorial radius at the critical limit. The critical velocity $v_{\text {crit, }, 1}$, which corresponds to the Keplerian velocity at the stellar surface, is the maximum rotational velocity a star can reach in hydrostatic equilibrium. For massive Population I (Pop I) stars, the critical limit constrains the accreted angular momentum to be less than one-third of the Keplerian angular momentum (Haemmerlé et al. 2017). If it exceeds this value, internal angular momentum redistribution by convection leads the stellar surface to rotate above the critical limit.

In the present work, we examine similar constraints from stellar evolution on the accretion of angular momentum in the case of Pop III SMSs. These stars evolve at nearly their Eddington limit, at which radiation pressure alone cancels gravity. However, this limit is not actually reached by SMSs, but is approached asymptotically as their masses grow. Departures from this limit are expressed by the Eddington factor,

$$
\Gamma_{\text {Edd }}:=\frac{d P_{\text {rad }}}{d r} /-\rho \frac{G M}{R^{2}},
$$

where $d P_{\text {rad }} / d r$ and $\rho$ are the radiation pressure gradient and the mass density at the photosphere, $G$ is the gravitational constant, and $M$ and $R$ are the stellar mass and radius, respectively. For a star close to the Eddington limit $\left(\Gamma_{\text {Edd }} \gtrsim 0.6\right)$, the critical velocity in Equation (1) must be replaced by

$$
\mathrm{v}_{\text {crit }, 2}^{2}=R_{\mathrm{eq}}^{2} \cdot 2 \pi G \bar{\rho} \cdot\left(1-\Gamma_{\mathrm{Edd}}\right),
$$




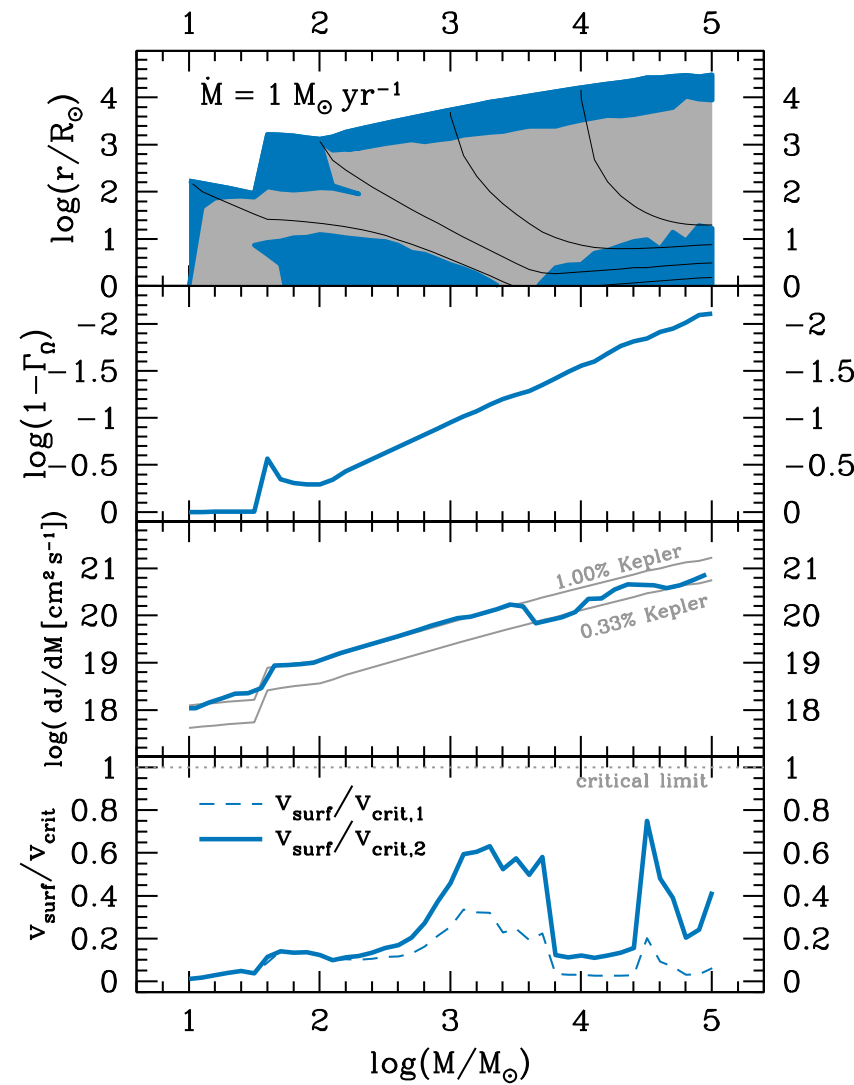

Figure 1. Internal structure, Eddington factor, accreted angular momentum, and surface velocity of the model with $\dot{M}=1 M_{\odot} \mathrm{yr}^{-1}$ and $j_{\mathrm{accr}} \simeq 0.01 j_{\mathrm{K}}$, as a function of the current stellar mass. In the top panel, the upper envelope is the photospheric radius, the colored regions are convective zones, the gray regions are radiative, and the black lines indicate the contracting Lagrangian layers of $\log \left(M_{r} / M_{\odot}\right)=1,2,3$, and 4 . The Eddington factor shown in the second panel is the corrected Eddington factor in the case with rotation (Equation (4)). In the third panel, the theoretical $j_{\text {accr }}$ for $f=1 \%$ and $0.33 \%$ is indicated by the gray lines, while the colored one is the actual angular momentum accreted by the model. In the bottom panel, the surface velocity is plotted as a ratio of the critical velocities $v_{\text {crit, } 1}$ and $v_{\text {crit, } 2}$ of Equations (1) and (3).

where again $R_{\text {eq }}$ is the equatorial radius and $\bar{\rho}$ the mean mass density of the star (Maeder \& Meynet 2000). This second critical velocity reflects the contribution from both the centrifugal force and the radiation pressure to counteract gravity. Similarly, in the presence of rotation, the Eddington factor defined in Equation (2) has to be replaced by

$$
\Gamma_{\Omega}=\frac{d P_{\mathrm{rad}}}{d r} / \rho\left(-\frac{G M}{R^{2}}+a_{c}\right)=\frac{d P_{\mathrm{rad}}}{d P}=\frac{\Gamma_{\mathrm{Edd}}}{1-\frac{\Omega^{2}}{2 \pi G \bar{\rho}}},
$$

where $a_{c}$ is the centrifugal acceleration, $P$ the total pressure, and $\Omega$ the angular velocity. This is called the $\Omega \Gamma$ limit (Maeder $\&$ Meynet 2000). Equation (3) shows that as $\Gamma_{\mathrm{Edd}} \rightarrow 1$, $v_{\text {crit }, 2} \rightarrow 0$. Previous works investigated the impact of the $\Omega \Gamma$ limit on Pop III stars (Yoon et al. 2015; Lee \& Yoon 2016), but SMSs were not considered.

Here, we present a model of an accreting Pop III SMS, following self-consistently its internal differential rotation, with the aim of constraining the angular momentum accreted by such objects and establishing their rotational properties. The stellar evolution code is described in Section 2 and the model in Section 3. We discuss our results in Section 4 and conclude in Section 5.

\section{Stellar Evolution Code}

The GENEVA code is a one-dimensional hydrostatic stellar evolution code that numerically solves the four structure equations (Eggenberger et al. 2008). Differential rotation is included, with the assumption of shellular rotation, according to which each isobar rotates as a solid body (Meynet \& Maeder 1997). Angular momentum transport between the various isobars includes three processes: convection, shear diffusion, and meridional circulation. Convective transport is treated by assuming solid-body rotation in each convective zone. In radiative regions, the equation of angular momentum transport by shear diffusion and meridional currents reads:

$$
\rho \frac{d\left(r^{2} \Omega\right)}{d t}=\frac{1}{5 r^{2}} \frac{d}{d r}\left(\rho r^{4} \Omega U(r)\right)+\frac{1}{r^{2}} \frac{d}{d r}\left(\rho D(r) r^{4} \frac{d \Omega}{d r}\right),
$$

where $\Omega$ is the angular velocity, $U(r)$ is the amplitude of the radial component of the meridional velocity, and $D(r)$ is the diffusion coefficient for shear instability (using the prescription of Maeder 1997). Accretion is included, at a rate $\dot{M}$ fixed externally, as a free parameter. The thermal properties of the accreted material are those of cold disk accretion (Palla \& Stahler 1992), and its rotational properties are fixed externally through the angular momentum we attribute to the new layers. In the presence of strong outward angular momentum transport in the external layers (e.g., a convective envelope), numerical instabilities prevent full control of the accreted angular momentum. The code also includes a GR correction to the equation of hydrostatic equilibrium, the first-order post-Newtonian Tolman-Oppenheimer-Volkoff correction (Fuller et al. 1986). A general description of the code with rotation, without accretion, can be found in Eggenberger et al. (2008). The treatment of accretion is detailed in Haemmerlé et al. (2016, 2017).

\section{Models}

\subsection{Inputs to the Models}

We consider the fiducial accretion rate

$$
\dot{M}=1 M_{\odot} \mathrm{yr}^{-1},
$$

typical of SMS formation (e.g., Latif et al. 2013b; Smidt et al. 2017). We give the accreted material a fixed fraction of the Keplerian angular momentum,

$$
j_{\text {accr }}:=\frac{d J}{d M}=f \cdot j_{\mathrm{K}} \quad \text { with } j_{\mathrm{K}}=\sqrt{G M R},
$$

where $J$ is the angular momentum of the star. We consider $f=0.01$, i.e., the angular momentum accreted is $1 \%$ of the Keplerian angular momentum.

For numerical stability, we start the run at a stellar mass of $10 M_{\odot}$. The initial model is fully convective (polytrope with $n \simeq 3 / 2$, flat entropy profile), with a radius and central temperature of $R=171 R_{\odot}$ and $T_{c}=4 \times 10^{5} \mathrm{~K}$, respectively. Because of convection, the initial model rotates as a solid body, whose rotation profile is fully determined by one parameter. We choose a surface velocity that corresponds to a fraction $f=0.01$ of the critical velocity (Equation (1)). The chemical compositions of the initial model and accreted material are 
identical $(X=0.7516, Y=0.2484$, and $Z=0)$. We stop the run at a stellar mass of $10^{5} M_{\odot}$ because of numerical instability.

\subsection{Rotational Properties of the Model}

Figure 1 shows the evolution of the internal structure, the Eddington factor, the accreted angular momentum, and the surface velocity (ratio to the critical velocities $v_{\text {crit, } 1}$ and $v_{\text {crit, } 2 \text { ) }}$ of the model with the inputs of Section 3.1. The internal structure shown here is identical to that described in Haemmerlé et al. (2018). The impact of rotation on the internal structure (as well as on the evolutionary track) is negligible. In the beginning of the evolution, a radiative core forms and grows in mass, enhancing the internal flux and producing a swelling of the radius by one order of magnitude (luminosity wave; Larson 1972). At such high accretion rates, the stellar surface cannot contract to the ZAMS, and the radius remains large $\left(\sim 10^{4} R_{\odot}\right)$, growing as $R \propto M^{1 / 2}$ as the evolution proceeds, despite the contraction of each Lagrangian layer. The star evolves along the Hayashi line, with a convective envelope due to the low temperature $\left(\sim 10^{4}-10^{5} \mathrm{~K}\right)$ in the inflated regions. In the center, after the layers corresponding to the initial model $\left(M_{r}<10 M_{\odot}\right)$ have significantly contracted (at $M \simeq 3000 M_{\odot}$ ), the temperature exceeds $10^{8} \mathrm{~K}$ and H-burning becomes efficient, triggering convection. This convective core grows in mass as the evolution proceeds.

Once the luminosity wave reaches the surface, the Eddington factor exceeds $50 \%$ and eventually evolves as

$$
\log \left(1-\Gamma_{\Omega}\right) \simeq 1-\frac{2}{3} \log \frac{M}{M_{\odot}} .
$$

It reaches $90 \%$ at $M=10^{3} M_{\odot}$ and $99 \%$ at $M=6.3 \times 10^{4} M_{\odot}$.

Until several hundred $M_{\odot}$, the rotation velocity remains small $\left(<20 \% v_{\text {crit, } 1}\right)$. But when the star approaches $1000 M_{\odot}$, the rapid contraction of the Lagrangian layers and the instantaneous $J$-transport outward in the convective envelope cause $v_{\text {surf }}$ to increase. When $v_{\text {surf }} / v_{\text {crit }, 2}>50 \%$, numerical convergence becomes difficult to achieve and we have to decrease $j_{\text {accr }}$ by a factor of 3 . Several oscillations occur between $j_{\text {accr }}=1 \%-0.33 \% j_{\mathrm{K}}$, leading to oscillations in $v_{\text {surf }} / v_{\text {crit, } 2}(10 \%-80 \%)$. The run stops at $M=10^{5} M_{\odot}$ with $v_{\text {surf }} / v_{\text {crit }, 1} \simeq 10 \%$ and $v_{\text {surf }} / v_{\text {crit }, 2} \simeq 40 \%$. Notice that the Eddington factor exceeds 0.6 at $M \simeq 100 M_{\odot}$, so that $v_{\text {surf }} / v_{\text {crit, } 2}>v_{\text {surf }} / v_{\text {crit, } 1}$ in later stages by a factor of a few. We emphasize that the oscillations in $j_{\text {accr }}$ are numerical (see Section 2). However, the actual angular momentum accretion history of an SMS is not expected to strictly follow $j_{\text {accr }}=f \cdot j_{\mathrm{K}}$ with a constant $f$, so that the presence of these oscillations does not affect the physical consistency of the model.

In order to see the effect of differential rotation in the model, we plot in Figure 2 the evolution of the angular velocity $\Omega$ in the center and at the surface as a function of the stellar mass. We also plot the evolution of $v_{\text {surf }} / v_{\text {crit, } 2}$ of our model, compared to the value obtained with the assumption of solidbody rotation. In the beginning of the evolution, the $\Omega$ gradient in the growing radiative core is slightly positive outward. But differential rotation remains small, and $\Omega$ increases in all the contracting layers, because of local angular momentum conservation. Once the swelling occurs, the internal contraction departs strongly from homology, and differential rotation develops in the large radiative region. Indeed, with local angular momentum conservation, one has $\dot{\Omega} / \Omega=-2 \dot{r} / r$, so

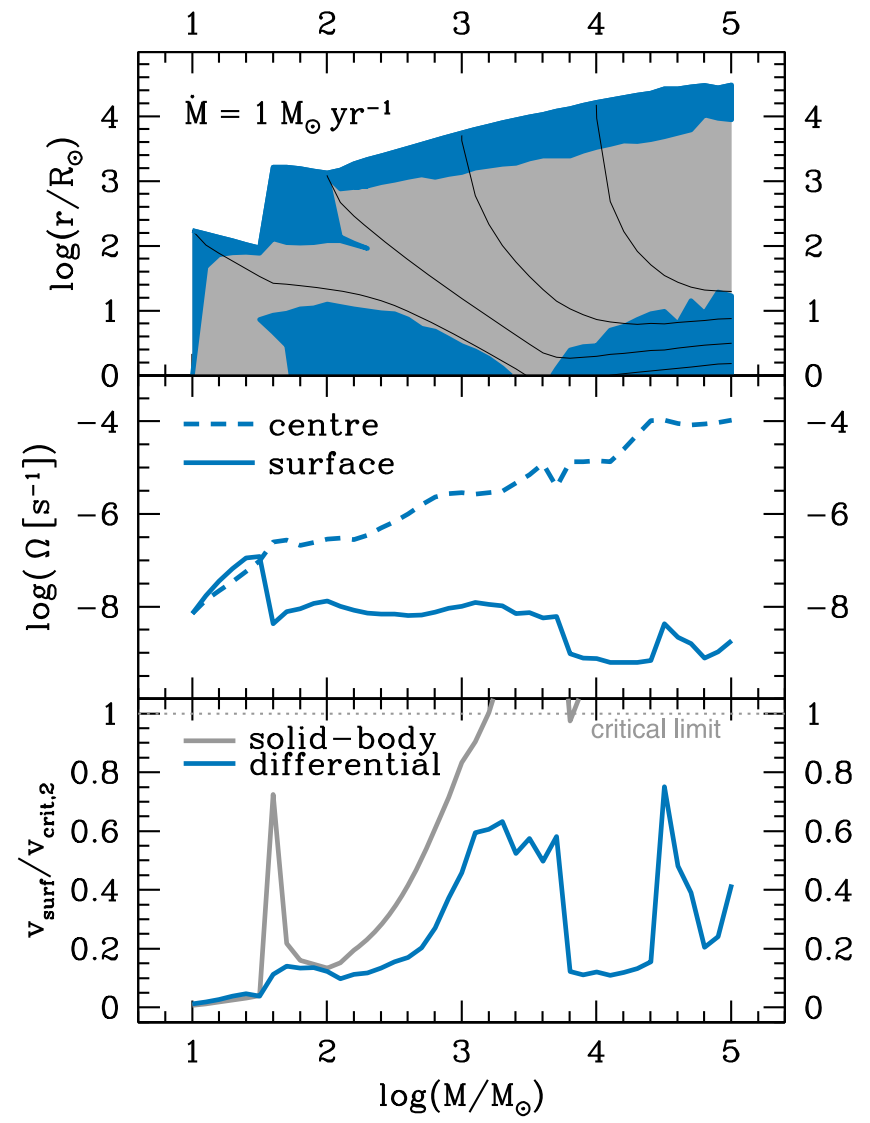

Figure 2. Differential rotation in the star, for the same model as Figure 1. The top panel shows again the stellar structure as a point of reference, and is the same as in Figure 1. The second panel shows the evolution of the angular velocity in the center and at the surface. The bottom panel indicates $v_{\text {surf }} / v_{\text {crit, } 2}$ of the model, with differential rotation (same as in Figure 1) and the $v_{\text {surf }} / v_{\text {crit, } 2}$ obtained if we assume solid-body rotation with the same $j_{\text {acr }}\left(\Omega_{\text {sol }}:=J / I\right.$, where $I$ is the total moment of inertia of the star).

that non-homology (i.e., $\dot{r} / r$ depends on $r$ ) enhances differential rotation $(\dot{\Omega} / \Omega$ and thus $\Omega(t)$ depend on $r)$. The angular velocity of the external layers decreases by more than one order of magnitude while that of the center stops growing temporarily due to the instantaneous $J$-transport in the transient convective core. This results in an $\Omega$ gradient that is strongly negative outward, in particular in the external layers that experience the swelling, where departures from homology are more pronounced. When the transient convective core stops growing in mass and contracts in radius with the Lagrangian layers, $\Omega$ increases again in the center. At the same time, the surface has converged to the Hayashi limit, and its angular velocity stays nearly constant $\left(\simeq 10^{-8} \mathrm{~s}^{-1}\right)$. Thus, differential rotation is enhanced by the central contraction and $\Omega$ soon differs by 3-4 orders of magnitude between the center and the surface. The spinning-up of the center stops only when H-burning becomes efficient enough to halt contraction in the convective core at 20,000-30,000 $M_{\odot}$. Then the ratio of $\Omega$ in the core and at the surface stabilizes at a level of 4-5 orders of magnitude, depending on the variations in $j_{\text {accr }}$.

Angular momentum transport by shear diffusion remains negligible during the whole evolution because of the short evolutionary timescale. This is also true for meridional circulation, except in a small region under the convective envelope. During the entire evolution, the internal angular 
momentum distribution is dominated by convective transport and local angular momentum conservation. Since large regions of the star are radiative and contraction is far from homology, differential rotation is extremely strong.

The impact of differential rotation on the evolution of the surface velocity is shown in the bottom panel of Figure 2. While for differential rotation $v_{\text {surf }} / v_{\text {crit,2 }}$ remains in the range $10 \%-80 \%$, the value of $v_{\text {surf }} / v_{\text {crit }, 2}$ computed by assuming rigid rotation reaches the critical limit before the star becomes supermassive. After a short peak at $70 \%$ during the swelling, this ratio falls to $10 \%$ and then grows rapidly due to the increase in $\Gamma_{\text {Edd }}$ (through the decrease in $v_{\text {crit,2}}$; Equation (3)). The $\Omega \Gamma$ limit is reached at $1500 M_{\odot}$, which is long before the star enters the supermassive regime.

\section{Discussion}

\subsection{Semi-analytical Interpretation}

If we neglect the deformation of the star $\left(R_{\mathrm{eq}}=R\right)$, which is justified for $v_{\text {surf }} / v_{\text {crit }, 1} \lesssim 50 \%$, the corrected critical velocity from Equation (3) can be written as

$$
v_{\text {crit }, 2}=\frac{3}{2} v_{\text {crit }, 1} \sqrt{1-\Gamma_{\text {Edd }}},
$$

since $\bar{\rho}=M_{r} / \frac{4}{3} \pi R^{3}$. The model described in Section 3.2 shows that the $\Gamma_{\text {Edd }}-M$ relation can be fitted by a power law (Equation (8)). Using this fit in Equation (9), one obtains

$$
\frac{v_{\text {crit }, 2}}{v_{\text {crit }, 1}} \simeq 4.7 \times\left(\frac{M}{M_{\odot}}\right)^{-1 / 3},
$$

which gives $v_{\text {crit }, 2} / v_{\text {crit }, 1}=22 \%$ for $\quad M=10^{4} \quad M_{\odot}$ and $v_{\text {crit }, 2} / v_{\text {crit }, 1}=10 \%$ for $M=10^{5} M_{\odot}$. Thus, an SMS has to rotate at less than $10 \%-20 \%$ of its classical critical velocity $v_{\text {crit, } 1}$, otherwise it would exceed $v_{\text {crit, } 2}$ and would reach the $\Omega \Gamma$ limit. This is in agreement with the model in Figure 1. We illustrate this semi-analytical interpretation in Figure 3.

\subsection{Constraints on the Accretion of Angular Momentum}

The model described above shows that for accreting SMSs to avoid the $\Omega \Gamma$ limit their accreted angular momentum must be of the order of $1 \%$ of the Keplerian angular momentum. It indicates that SMS formation by accretion requires strong mechanisms for extracting angular momentum from the accretion disk-like magnetic fields (Schober et al. 2012; Latif \& Schleicher 2016), viscosity (Popham \& Narayan 1991; Takahashi \& Omukai 2017), or gravitational torques (Wise et al. 2008). This is a particular case of the classical angular momentum problem (Spitzer 1978; Bodenheimer 1995; Maeder 2009). The constraint obtained here is much stronger than in the case of massive Pop I stars $\left(j_{\text {accr }} \lesssim 1 / 3 \quad j_{\mathrm{K}}\right.$; Haemmerlé et al. 2017). Notice that the constraint from the disk itself, i.e., sub-Keplerian rotation, already implies a loss of angular momentum in the accretion disk. The constraint from the star obtained here corresponds to an additional angular momentum loss of $99 \%$ of the Keplerian angular momentum at the stellar surface. The rate of this additional angular momentum loss can be estimated by $\dot{J}_{\Omega \Gamma}=\dot{M} j_{\mathrm{K}}(1-f) \simeq \dot{M} \sqrt{G M R}$. In the supermassive regime, this gives $\dot{J}_{\Omega \Gamma} \simeq 10^{48}-10^{49} \mathrm{~g} \mathrm{~cm}^{2} \mathrm{~s}^{-2}$. In the timescale of the

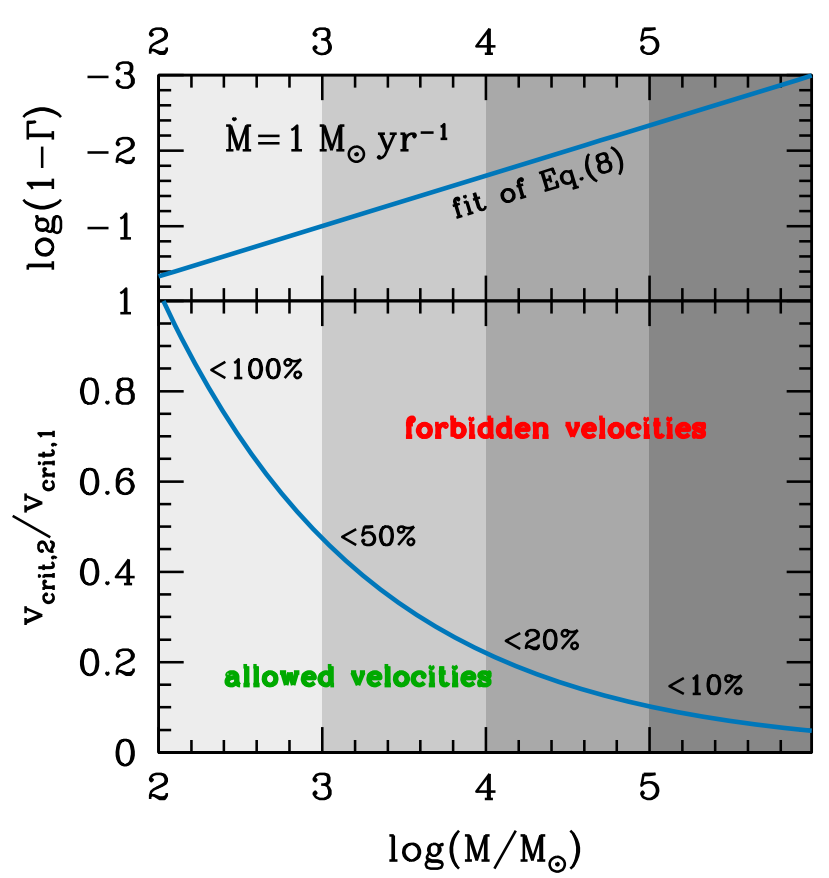

Figure 3. Semi-analytical interpretation of the constraint from the $\Omega \Gamma$ limit. The top panel shows the fit of Equation (8). The bottom panel shows the resulting $v_{\text {crit, } 2} / v_{\text {crit, } 1}$ vs. $M$ relation (Equation (10)) that delimits the allowed and forbidden surface rotation velocities.

evolution $\left(10^{4}-10^{5}\right.$ years), the star-disk system has to lose $\sim 10^{60} \mathrm{~g} \mathrm{~cm}^{2} \mathrm{~s}^{-1}$ in addition to the losses required to maintain sub-Keplerian rotation in the disk.

On the other hand, the effect of differential rotation softens the constraint on $j_{\text {accr }}$. Assuming solid-body rotation, Lee \& Yoon (2016) computed the rotational properties of Pop III stars accreting at $\dot{M} \sim 10^{-3} M_{\odot} \mathrm{yr}^{-1}$. They found that, even for $j_{\text {accr }}=0.01 j_{\mathrm{K}}$, the $\Omega \Gamma$ limit is reached at $M=20-40 M_{\odot}$, preventing more massive stars from forming by accretion. Due to the differences in the initial conditions and the accretion rates, a direct comparison of the early evolution is not possible. However, our model shows that solid-body rotation requires $j_{\text {accr }} \ll 0.01 j_{\mathrm{K}}$ for the star to become supermassive by accretion, while with differential rotation $j_{\mathrm{accr}}=0.01 j_{\mathrm{K}}$ is low enough. This suggests that the constraint on $j_{\text {accr }}$ obtained by Lee \& Yoon (2016) is artificially strong due to their assumption of solid-body rotation.

What happens once the star reaches the $\Omega \Gamma$ limit? According to the stationary disk models of Takahashi \& Omukai (2017), viscosity is able to maintain the advection of angular momentum arbitrarily low. Their models show that stationary solutions exist for any $M$ and $\dot{M}$ with a $j_{\text {accr }}$ as close to 0 as required, provided that the surface density in the inner disk is high enough. Thus, if at a given point the accretion process is stopped by the $\Omega \Gamma$ limit, the mass that rotates too fast will accumulate in the disk until viscosity is efficient enough to extract the excess of angular momentum. If this picture is correct, the star-disk system will adjust to a configuration corresponding to the highest $j_{\text {accr }}$ compatible with the $\Omega \Gamma$ limit. Our model can then be seen as a lower limit for $j_{\text {accr }}$ and $v_{\text {surf }}$ because $v_{\text {surf }} / v_{\text {crit,2 }}$ always remains lower than $80 \%$. 


\section{Conclusion}

We have described the first stellar evolution model of Pop III SMS that simultaneously include accretion and rotation. We obtained the following results:

1. SMSs have to be slow rotators. Since SMSs evolve at close to the Eddington limit, the $\Omega \Gamma$ limit imposes tight constraints on their rotation velocity. For $M>10^{4} M_{\odot}$, the rotation velocity cannot exceed $\sim 20 \%$ of the first critical velocity (Equation (1)). For $M>10^{5} M_{\odot}$, the limit is $\sim 10 \%$. For such slow rotators, the impact of rotation on the stellar structure is negligible.

2. SMS formation by accretion requires mechanisms efficient enough to remove most ( $\sim 99 \%)$ of the angular momentum from the accretion disk. Indeed, the constraint on the rotation velocity translates into a constraint on the accreted angular momentum, which must not exceed $1 \%$ of the Keplerian value. The main mechanisms expected to play this role are viscosity, magnetic fields, and gravitational torques from spiral arms in the accretion disk. If the angular momentum accreted remains slightly lower than $1 \%$ of the Keplerian angular momentum, then accretion can proceed toward $M>10^{5} M_{\odot}$ without facing the $\Omega \Gamma$ limit.

3. SMSs forming by accretion rotate highly differentially, with a frequency in the core 4 or 5 orders of magnitude higher than in the envelope. This is due to the highly nonhomologous nature of internal stellar contraction. In radiative regions, the rotation profile is dominated by local angular momentum conservation, so that departures from homology result in departures from solid-body rotation. Differential rotation softens the constraint on the accreted angular momentum because it allows more angular momentum to be contained in the central regions while keeping a slow rotation velocity at the surface.

L.H. and R.S.K. were supported by the European Research Council under the European Community's Seventh Framework Programme (FP7/2007-2013) via the ERC Advanced Grant "STARLIGHT: Formation of the First Stars" (project number 339177). This work was sponsored by the Swiss National Science Foundation (project number 200020-172505). A.H. was supported by an ARC Future Fellowship (FT120100363) and received partial support from the US National Science Foundation under grant No. PHY-1430152 (JINA Center for the Evolution of the Elements). D.J.W. was supported by STFC New Applicant Grant ST/P000509/1.

\section{ORCID iDs}

Lionel Haemmerlé (i) https://orcid.org/0000-0001-5614-5493 Ralf S. Klessen iㅏ https://orcid.org/0000-0002-0560-3172
Alexander Heger (1) https://orcid.org/0000-0002-3684-1325

Daniel J. Whalen iㅏ https://orcid.org/0000-0001-6646-2337

\section{References}

Agarwal, B., Khochfar, S., Johnson, J. L., et al. 2012, MNRAS, 425, 2854 Agarwal, B., Regan, J., Klessen, R. S., Downes, T. P., \& Zackrisson, E. 2017, MNRAS, 470, 4034

Becerra, F., Greif, T. H., Springel, V., \& Hernquist, L. E. 2015, MNRAS, 446, 2380

Begelman, M. C., Volonteri, M., \& Rees, M. J. 2006, MNRAS, 370, 289

Bodenheimer, P. 1995, ARA\&A, 33, 199

Bromm, V., \& Loeb, A. 2003, ApJ, 596, 34

Chandrasekhar, S. 1964, ApJ, 140, 417

Dijkstra, M., Ferrara, A., \& Mesinger, A. 2014, MNRAS, 442, 2036

Eggenberger, P., Meynet, G., Maeder, A., et al. 2008, Ap\&SS, 316, 43

Fuller, G. M., Woosley, S. E., \& Weaver, T. A. 1986, ApJ, 307, 675

Haemmerlé, L., Eggenberger, P., Meynet, G., et al. 2017, A\&A, 602, A17

Haemmerlé, L., Eggenberger, P., Meynet, G., Maeder, A., \& Charbonnel, C. 2016, A\&A, 585, A65

Haemmerlé, L., Woods, T. E., Klessen, R. S., Heger, A., \& Whalen, D. J. 2018, MNRAS, 474, 2757

Hirano, S., Hosokawa, T., Yoshida, N., \& Kuiper, R. 2017, Sci, 357, 1375

Hosokawa, T., Omukai, K., \& Yorke, H. W. 2012, ApJ, 756, 93

Hosokawa, T., Yorke, H. W., Inayoshi, K., Omukai, K., \& Yoshida, N. 2013, ApJ, 778, 178

Inayoshi, K., Hosokawa, T., \& Omukai, K. 2013a, MNRAS, 431, 3036

Inayoshi, K., Omukai, K., \& Tasker, E. 2014, MNRAS, 445, L109

Inayoshi, K., Sugiyama, K., Hosokawa, T., Motogi, K., \& Tanaka, K. E. I. 2013b, ApJL, 769, L20

Larson, R. B. 1972, MNRAS, 157, 121

Latif, M. A., \& Schleicher, D. R. G. 2016, A\&A, 585, A151

Latif, M. A., Schleicher, D. R. G., Schmidt, W., \& Niemeyer, J. 2013a, MNRAS, 433, 1607

Latif, M. A., Schleicher, D. R. G., Schmidt, W., \& Niemeyer, J. C. 2013b, MNRAS, 436, 2989

Lee, H., \& Yoon, S.-C. 2016, ApJ, 820, 135

Maeder, A. 1997, A\&A, 321, 134

Maeder, A. 2009, Physics, Formation and Evolution of Rotating Stars (Berlin: Springer)

Maeder, A., \& Meynet, G. 2000, A\&A, 361, 159

Meynet, G., \& Maeder, A. 1997, A\&A, 321, 465

Palla, F., \& Stahler, S. W. 1992, ApJ, 392, 667

Popham, R., \& Narayan, R. 1991, ApJ, 370, 604

Regan, J. A., \& Haehnelt, M. G. 2009, MNRAS, 396, 343

Regan, J. A., Johansson, P. H., \& Wise, J. H. 2014, ApJ, 795, 137

Schober, J., Schleicher, D., Federrath, C., et al. 2012, ApJ, 754, 99

Smidt, J., Whalen, D. J., Johnson, J. L., \& Li, H. 2017, ApJ, submitted (arXiv:1703.00449)

Spitzer, L. 1978, Physical Processes in the Interstellar Medium (New York: Wiley-Inter Science)

Stacy, A., Bromm, V., \& Loeb, A. 2011, MNRAS, 413, 543

Stacy, A., Greif, T. H., Klessen, R. S., Bromm, V., \& Loeb, A. 2013, MNRAS, 431,1470

Takahashi, S. Z., \& Omukai, K. 2017, MNRAS, 472, 532

Umeda, H., Hosokawa, T., Omukai, K., \& Yoshida, N. 2016, ApJL, 830, L34

Wise, J. H., Turk, M. J., \& Abel, T. 2008, ApJ, 682, 745

Woods, T. E., Heger, A., Whalen, D. J., Haemmerlé, L., \& Klessen, R. S. 2017, ApJL, 842, L6

Yoon, S.-C., Kang, J., \& Kozyreva, A. 2015, ApJ, 802, 16 